\title{
Nomogram predicted overall survival of patients with neuroendocrine carcinoma of the breast
}

\author{
Wenwen Tian ( $\square$ tianww@sysucc.org.cn ) \\ Sun Yat-sen University Cancer Center https://orcid.org/0000-0003-2701-5993 \\ Xinhua Xie \\ Sun Yat-sen University Cancer Center \\ Yanan Kong \\ Sun Yat-sen University Cancer Center \\ Peng Liu \\ Sun Yat-sen University Cancer Center \\ Weige Tan \\ The First Affliated Hospital of Guangzhou Medical University \\ Xiaoming Xie \\ Sun Yat-sen University Cancer Center
}

\section{Research}

Keywords: Neuroendocrine carcinoma of the breast, nomogram, prognosis, overall survival

Posted Date: February 19th, 2020

DOl: https://doi.org/10.21203/rs.2.23950/v1

License: (c) (i) This work is licensed under a Creative Commons Attribution 4.0 International License. Read Full License 


\section{Abstract}

Background For primary neuroendocrine carcinoma of breast was a very rare subtype in breast cancers, its prognosis was still controversial and there was no independent standard for its treatment. The purpose of our retrospective study was to construct a nomogram to predict the overall survival (OS) of patients with neuroendocrine carcinoma of the breast.

Methods 150 patients of training cohort were collected from Surveillance, Epidemiology, and End Results (SEER) database diagnosed between 2003 and 2015, and 93 patients of verification cohort were enrolled from Sun Yat-sen University Cancer Center (Guangzhou, China) diagnosed between 2004 and 2018. The nomogram was constructed uniting three significantly risk factors of overall survival identified by univariate and multivariate analysis and then validated using receiver operating characteristic (ROC) curves for discrimination, calibration plots and the decision curves analysis (DCA).

Results Age, N stage and PR status were closely and significantly related to overall survival in patients with breast neuroendocrine carcinoma. The $\mathrm{C}$-index of nomogram in the training and verification cohorts are $0.775(95 \% \mathrm{Cl}, 0.784$ to 0.615$)$ and $0.760(95 \% \mathrm{Cl}, 0.705$ to 0.800$)$ respectively. Calibration plots of practical and predicted possibility for the nomogram demonstrated that the predictive 5-year overall survival rate was in accordance with the actual overall survival probability in both sets. Moreover, the decision curves (DCA) also expressed pretty clinical benefit of the nomogram across a range of high-risk threshold.

Conclusion This novel population-based nomogram may help with treatment decisions in patients with neuroendocrine carcinoma of the breast (NEBC).

\section{Background}

Breast cancer was the number one multifactorial cancer in women worldwide,composed of many different subtypes with various clinicopathologic features. Neuroendocrine carcinoma of the breast is a rare special subtype, endorsed by the WHO for the first time in 2003[1]. Neuroendocrine tumors of the breast (NEBC) are very uncommon malignant tumors having a proportion of less than one percent in breast cancers[2]. Exactly as a result of its scarce, the existing learning of NECB is confined to clinical case reports and some small series analysis[3-18]. And most of the current knowledge concerning the disease was obtained from these studies. For specific recommendations regarding treatment do not exist so far, surgical management, similar to conventional breast cancer, was performed with NEBC patients[19]. Although curative surgical treatment has been performed, the long-term postoperative prognosis of NECB is still controversial.

On the prognosis, no consensus has been reached for NEBC. Contradictory research results appeared in the previous studies, very likely thanks to the limited reported number of patients, and different selection criteria[20]. Thus, identification of prognostic factors of NEBC has long been receiving much attention. And to get to know more about mammary NEC, we have made full use of a relatively complete and large 
cancer database collected over the past two decades from Surveillance, Epidemiology, and End Results (SEER) registries and Sun Yat-sen University Cancer Center (Guangzhou, China). Using these data, we assessed the onset and clinical prognosis of NEBC.

There were three goals of our present study. First, our aim is to assess the prognostic value of clinicopathological characteristics in NEBC patients. Secondly, we designed to establish a predictive nomogram which has been wildly used in many other cancer diseases to accomplish overall survival estimation for NEBC patients[21-27]. Finally, we aimed to evaluate the clinical applicability of the nomogram in predicting prognosis.

\section{Methods}

\section{Patients}

Two independent groups of NEBC patients after radically surgical resection were selected for this study. The training cohort patients $(n=150)$ were from Surveillance, Epidemiology, and End Results (SEER) registries diagnosed with NEBC between 2003 to 2015 and the verification cohort were collected from patients $(n=93)$ who received curative surgery in Sun Yat-sen University Cancer Center (Guangzhou, China) between 2004 and 2018. All patients had survived at least 3 months after radical surgery. Criteria for the inclusion and exclusion of patients analyzed in both cohorts are as follows: (a) confirmed pathological diagnosis of NEBC; (b) radically surgical resection of tumors with axillary lymph node dissection or intraoperative sentinel lymph node biopsy as well as no residual cancer; (c) the entire clinicopathologic and follow-up data were recorded; (d) the patients, who had no regional lymph node status and tumor size records were excluded from the analysis. Data announced from the SEER database hasn't demanded any patient consent because cancer was a disease that could be reported everywhere in the United States. For patients used for verification in the study, we have got informed consent from themselves and the research program has been approved by the Clinical Research Ethics Committee of Sun Yat-sen University Cancer Center. Clinicopathologic features analyzed included age, primary site, laterality, grade, axillary lymph node metastasis, T stage, N stage, ER status, PR status and HER2 status. In addition, we reviewed the outpatient visit records or contacted the patients by phone to determine the overall survival time, which was defined as the period between the time of surgery and time of either death or last follow-up,

\section{Statistical Analysis}

SPSS software (version 24.0) and R (version 3.4.4) were applied to statistical analyses of data. According to conventional clinical findings, the grouping of categorical variables was shown in Table 1. Univariate analysis was carried out to evaluate the statistical significance of clinical and pathological features. Multivariate analysis results were obtained by Cox proportional hazard regression. All the analyzed variables with $P$ values less than 0.05 in the multivariate analysis were used to create a predictive nomogram. The nomogram was established in the training set and then verified in the other validation set. The discrimination was quantified by the area under the receiver operating characteristic (ROC) 
curves (AUC or C-index). Graphically evaluation of calibration was executed by using the Hosmer fitting optimization test to draw the relationship between actually observed probabilities and predictive probabilities[28]. The Decision Curve Analysis (DCA) was applied to assess the clinical effectiveness of nomogram for the prognosis prediction. All tests were performed in both sets of queues and $\mathrm{P}<0.05$ was considered significant. 
Table 1

Basal clinicopathologic characteristics in training and verification cohort.

\section{Characteristics}

Age

$\leq 50$

$>50$

Primary Site

Central portion

UIQ

LIQ

UOQ

LOQ

Others

Laterality

Left

Right

Grade

I

II

III

Unknown

T Stage

T1

T2

T3

Regional lymph nodes
Training set

$n=150(\%)$
Verification set

$n=93(\%)$
$33(22.0 \%)$

117(78.0\%)

$4(2.7 \%)$

17(11.3\%)

$9(6.0 \%)$

49(32.7\%)

14(9.7\%)

$57(38.0 \%)$

63(42.0\%)

$87(58.0 \%)$

$46(49.5 \%)$

$47(50.5 \%)$

23(15.3\%)

9(9.7\%)

$27(29.0 \%)$

$13(14.0 \%)$

$13(14.0 \%)$

$39(41.9 \%)$

$54(58.1 \%)$

$2(2.2 \%)$

$29(31.2 \%)$

\begin{tabular}{|c|c|c|}
\hline Characteristics & $\begin{array}{l}\text { Training set } \\
n=150(\%)\end{array}$ & $\begin{array}{l}\text { Verification set } \\
n=93(\%)\end{array}$ \\
\hline \multicolumn{3}{|l|}{ Age } \\
\hline$\leq 50$ & $33(22.0 \%)$ & $39(41.9 \%)$ \\
\hline$>50$ & 117(78.0\%) & $54(58.1 \%)$ \\
\hline \multicolumn{3}{|l|}{ Primary Site } \\
\hline Central portion & $4(2.7 \%)$ & $2(2.2 \%)$ \\
\hline UIQ & $17(11.3 \%)$ & $29(31.2 \%)$ \\
\hline LIQ & $9(6.0 \%)$ & $13(14.0 \%)$ \\
\hline UOQ & $49(32.7 \%)$ & $13(14.0 \%)$ \\
\hline LOQ & $14(9.7 \%)$ & $9(9.7 \%)$ \\
\hline Others & $57(38.0 \%)$ & $27(29.0 \%)$ \\
\hline \multicolumn{3}{|l|}{ Laterality } \\
\hline Left & $63(42.0 \%)$ & $46(49.5 \%)$ \\
\hline Right & $87(58.0 \%)$ & $47(50.5 \%)$ \\
\hline \multicolumn{3}{|l|}{ Grade } \\
\hline I & $23(15.3 \%)$ & $3(3.2 \%)$ \\
\hline ॥ & $51(34.0 \%)$ & $31(33.3 \%)$ \\
\hline III & $76(50.7 \%)$ & $12(12.9 \%)$ \\
\hline Unknown & - & $47(50.5 \%)$ \\
\hline \multicolumn{3}{|l|}{ T Stage } \\
\hline $\mathrm{T} 1$ & $63(42.0 \%)$ & $40(43.0 \%)$ \\
\hline $\mathrm{T} 2$ & $70(46.7 \%)$ & $51(54.8 \%)$ \\
\hline T3 & 17(11.3\%) & $2(2.2 \%)$ \\
\hline
\end{tabular}

UIQ, upper-inner quadrant; LIQ, lower-inner quadrant; UOQ, upper- outer quadrant; LOQ, lower-outer quadrant; ER, estrogen receptor; PR, progesterone receptor; HER2, human epidermal growth factor receptor 2. 


\begin{tabular}{|c|c|c|}
\hline Characteristics & $\begin{array}{l}\text { Training set } \\
n=150(\%)\end{array}$ & $\begin{array}{l}\text { Verification set } \\
n=93(\%)\end{array}$ \\
\hline Positive & $54(36.0 \%)$ & $24(25.8 \%)$ \\
\hline Negative & $96(64.0 \%)$ & $69(74.2 \%)$ \\
\hline \multicolumn{3}{|l|}{ N Stage } \\
\hline NO & $95(63.3 \%)$ & $69(74.2 \%)$ \\
\hline N1 & $38(25.3 \%)$ & $17(18.3 \%)$ \\
\hline N2 & $8(5.3 \%)$ & $5(5.4 \%)$ \\
\hline N3 & $8(5.3 \%)$ & $2(2.2 \%)$ \\
\hline \multicolumn{3}{|l|}{ ER status } \\
\hline Positive & 114(76.0\%) & $89(95.7 \%)$ \\
\hline Negative & $35(23.3 \%)$ & $2(2.2 \%)$ \\
\hline Unknown & $1(0.7 \%)$ & $2(2.2 \%)$ \\
\hline \multicolumn{3}{|l|}{ PR status } \\
\hline Positive & $92(61.3 \%)$ & $84(90.3 \%)$ \\
\hline Negative & $57(38.0 \%)$ & $9(9.7 \%)$ \\
\hline Unknown & $1(0.7 \%)$ & - \\
\hline \multicolumn{3}{|l|}{ Her 2 status } \\
\hline Positive & $2(1.3 \%)$ & $5(5.4 \%)$ \\
\hline Negative & $76(50.7 \%)$ & $81(87.1 \%)$ \\
\hline Unknown & $72(48.0 \%)$ & $7(7.5 \%)$ \\
\hline
\end{tabular}

\section{Results}

\section{Clinicopathological characteristics}

The comprehensive characteristics of all enrolled patients in the training and verification cohorts were shown in Table 1; the median survival time for the two groups were 35.5 months and 30 months, respectively. The median age of onset in the two sets were 63 years (range, 34-99 years) and 54 years (range, 25-85 years), respectively. The location of neuroendocrine breast carcinoma lesions were on the left breast in 109 cases (44.86\%) and on the right breast in 134 cases (55.14\%). For typical immune 
indicators, most patients were negative for axillary lymph node metastasis, positive for ER and PR status and negative for HER2 status in both sets. No significant differences were found between the training and verification groups with regard to the above-mentioned clinicopathologic characteristics.

\section{Identification of prognostic factors in patients with NEBC}

To identify potential prognostic factors, we performed univariate analysis on the training group with the result indicating in Table 2. Univariate analysis revealed that age $(p<0.001)$, axillary lymph node metastasis $(\mathrm{N}$ stage) $(p<0.001)$ and PR status $(p=0.009)$ were confirmed as the most important prognostic factors for OS. In multivariate analysis, age (hazard ratio [HR], 1.052; 95\% confidence interval $[\mathrm{Cl}], 1.024-1.082 ; \mathrm{p}<.001), \mathrm{N}$ stage $(\mathrm{HR}, 1.842 ; 95 \% \mathrm{Cl}, 1.270-2.673 ; \mathrm{p}=.001)$, and PR status (HR,5.157; $95 \% \mathrm{Cl}, 2.514-10.577 ; \mathrm{p}<.001$ ) were still significantly predictive factors of OS (Table 2 ). According to the above analysis results, we determined that age, $\mathrm{N}$ stage and PR status were independent factors affecting the OS of patients with NEBC, and then were integrated in the construction of nomogram. 
Table 2

Independent prognostic factors predicting OS in the training cohort.

\begin{tabular}{|c|c|c|}
\hline \multirow[t]{2}{*}{ Variables } & $\begin{array}{l}\text { Univariate analysis for } \\
\text { OS }\end{array}$ & $\begin{array}{l}\text { Multivariate analysis for } \\
\text { OS }\end{array}$ \\
\hline & HR $95 \% \mathrm{Cl} p$ value & $\begin{array}{l}\mathrm{HR} 95 \% \mathrm{Cl} \mathrm{p} \\
\text { value }\end{array}$ \\
\hline Age, years & $1.0531 .024-1.0830 .000$ & $1.0521 .024-1.0820 .000$ \\
\hline Primary Site & $1.0510 .929-1.1890 .431$ & NA \\
\hline Laterality (left vs right) & $1.2440 .658-2.3480 .502$ & NA \\
\hline Grade (I, II vs III) & $1.7500 .920-3.3270 .088$ & NA \\
\hline T Stage & 0.065 & NA \\
\hline T1 & 1.00 & \\
\hline $\mathrm{T} 2$ & $1.4720 .737-2.9380 .273$ & \\
\hline T3 & $2.4500 .941-6.3800 .067$ & \\
\hline $\begin{array}{l}\text { Regional lymph nodes (positive vs } \\
\text { negative) }\end{array}$ & $1.0110 .982-1.0400 .462$ & NA \\
\hline \multicolumn{3}{|l|}{ N Stage } \\
\hline NO & 1.000 .001 & 10.001 \\
\hline N1 & $0.8560 .366-2.0040 .720$ & $1.0420 .444-2.4460 .925$ \\
\hline N2 & $\begin{array}{l}3.4161 .163-10.029 \\
0.025\end{array}$ & $2.0190 .656-6.7780 .210$ \\
\hline N3 & $\begin{array}{l}6.3322 .314-17.326 \\
0.000\end{array}$ & $9.8013 .179-30.2170 .000$ \\
\hline ER status (positive vs negative) & $1.8590 .960-3.6020 .066$ & NA \\
\hline PR status (positive vs negative) & $2.3761 .241-4.5490 .009$ & $5.1572 .514-10.5770 .000$ \\
\hline Her 2 (positive vs negative) & $1.0520 .940-1.1760 .377$ & NA \\
\hline
\end{tabular}

\section{Construction and verification of prognostic nomogram for OS}

All the three independently predictive factors of OS in multivariate cox proportional hazards regression analysis derived from the training cohort were integrated into the prediction nomogram model which is 
shown in Fig. 1. The nomogram demonstrated good accuracy with an unadjusted concordance index (Cindex) of $0.775(95 \% \mathrm{Cl}, 0.784$ to 0.615$)$ (Fig. $2 \mathrm{~A})$. The calibration curve of survival probability for 5 years after operation also showed pretty good consistency between the nomogram model and actual observation prediction (Fig. 2C).

In the verification cohort, the median follow-up time was 30 months (range, 3-169 months). The C-index of the constructed nomogram for predicting 5-year OS was $0.760(95 \% \mathrm{Cl}, 0.705$ to 0.800$)$ (Fig. 2B), and good calibration was observed for predicting the probability of 5-year OS (Fig. 2D).

\section{Clinical usefulness of the nomogram as evaluated by DCA}

Given that the established nomogram demonstrated good predictive capabilities in terms of C-index, DCA was needed to determine the clinical validity of the nomogram. On DCA, the nomogram showed great net benefit with a wide range of threshold probability (Fig. 3A). Then we performed DCA in the verification cohort which also presents a similar wide range of threshold probability (Fig. 3B). This result further demonstrated fairly good estimation of clinical decision making at high net benefit levels.

\section{Discussion}

Mammary NEC has always been a controversial disease. Different studies have conveyed different clinical results owing to inconsistent diagnostic criteria in part, Some have revealed that the prognosis of neuroendocrine carcinomas was better than that of patients without specific types of aggressive cancer, whereas a worse prognosis has recently been observed in more larger series $[9,14,16,29]$. To study more about prognosis of NEBC, we collected total 243 NEBC patients who underwent curative surgery both from the SEER and Sun Yat-sen University Cancer Center (Guangzhou, China) database and constructed two groups, the training cohort and verification cohort, respectively. On the basis of the two separate queues, novel and effective nomogram have been developed to project the individual probability of overall survival at 5 years. With integration of the independent prognostic factors that reflect clinical points and postoperative pathological results, the nomogram was expected to be an effective and reliable tool.

Most population-based results showed that NECB is often positive for hormone receptors, whereas HER2 is almost always negative. In agreement with these previous series of study, our results also showed that the positive rate of ER, PR and HER2 are $83.5 \%, 72.4 \%$ and $2.9 \%$ respectively. Meanwhile, the study also presented that NEBC tended to occur in elderly patients (mean age 59 years), showing a higher pathological grade, a larger tumor (mean $27 \mathrm{~mm}$ ) and less local lymph node metastasis.

The advantage of our present study was that two cohorts of NEBC patients were enrolled for analysis, and they all underwent radically surgical treatment. Then major confounding factors for clinical outcomes of NEBC, including age, $\mathrm{N}$ stage and PR status, were eliminated. The nomogram possessed Cindices of 0.775 and 0.760 for 5-year OS in the training and verification cohort separately; although not perfect, the widely used nomogram was first applied in mammary neuroendocrine carcinoma to predict postoperative survival. Besides, the nomogram was developed in the training set and then validated in the 
other verification set to get more reliability. This tool could further promote individualized clinical decisions by applying clinically pathological factors that were readily available to estimate the overall survival in patients with NEBC.

Although the nomogram showed good accuracy for predicting postoperative overall survival, we must consider some limitations of this study. First of all, due to the lack of postoperative recurrence follow-up, we did not incorporate DFS into the study. Hence, postoperative disease-free survival of NEBC patients couldn't be predicted via the nomogram. Secondly, the nomogram was clinically suitable for postoperative decision-making, but not for preoperative decision making. Thirdly, tumor stage and grade should be considered as risk factors; however, in univariate analysis, they both showed no statistical difference. This may hinder the ability of the nomogram to predict prognosis. Finally, the possibility of selective bias was introduced because of the use of retrospective data. And patients of the training cohort were white, so estimation for non-white patients might not be as accurate as prediction. Given these above, we should conduct a larger sample size and longer follow-up study to further confirm the accuracy of the nomogram.

\section{Conclusion}

The novel population-based nomogram, developed from retrospectively collected data, could precisely predict 5-year OS of patients with neuroendocrine carcinoma of breast and thus help to make individualized treatment decisions. Definitely, larger queues or forward-looking studies were expected to further confirm our findings.

\section{Abbreviations}

NEBC

neuroendocrine carcinoma of the breast

C-index

concordance index

SEER

Surveillance, Epidemiology, and End Results

OS

overall survival

ROC

receiver operating characteristic

DCA

decision curves analysis

\section{Declarations}

\section{Ethics approval and consent to participate}


Data announced from the SEER database hasn't demanded any patient consent because cancer was a disease that could be reported everywhere in the United States. For patients used for verification in the study, we have got informed consent from themselves and the research program has been approved by the Clinical Research Ethics Committee of Sun Yat-sen University Cancer Center (the committee approval number GZR2016-076).

\section{Patient consent for publication}

Not applicable.

\section{Availability of data and materials}

The datasets used in our study are available from the author on reasonable request.

\section{Competing interests}

No potential conflict of interest.

\section{Funding}

The study was supported by funds from the National Natural Science Foundation of China (81302318 to Xinhua Xie; 81672598 to Xiaoming Xie), the Science and Technology Planning Projects of Guangdong (2017A030313554 to Weige Tan; 2017A020215163 to Xinhua Xie).

\section{Authors' contributions}

WT and XX designed the study and performed statistical analysis of the data. XX, YK and PL conducted the data analysis and interpretation. WT wrote the manuscript. Xiao. $X$ and Wei. Tan edited and revised the manuscript. All authors discussed the results.

\section{Acknowledgements}

Not applicable.

\section{References}

1. Bocker, W. 2002.[WHO classification of breast tumors and tumors of the female genital organs: pathology and genetics]. Verh Dtsch Ges Pathol. 86: 116-9.

2. Yang, W.T. and X.Z. Zhu. 2013.[The introduction of $2012 \mathrm{WHO}$ classification of tumours of the breast]. Zhonghua Bing Li Xue Za Zhi. 42: 78-80.

3. Lavigne, M., E. Menet, J.C. Tille, M. Lae, L. Fuhrmann, and C. Bonneau, et al. 2018.Comprehensive clinical and molecular analyses of neuroendocrine carcinomas of the breast. Mod Pathol. 31: 68-82.

4. Yang, X., Y. Cao, C. Chen, L. Liu, C. Wang, and S. Liu. 2017.Primary neuroendocrine breast carcinomas: a retrospective analysis and review of literature. Onco Targets Ther. 10: 397-407. 
5. Roininen, N., S. Takala, K.M. Haapasaari, A. Jukkola-Vuorinen, J. Mattson, and P. Heikkila, et al. 2017.Primary neuroendocrine breast carcinomas are associated with poor local control despite favourable biological profile: a retrospective clinical study. BMC Cancer. 17: 72.

6. Bogina, G., E. Munari, M. Brunelli, L. Bortesi, M. Marconi, and M. Sommaggio, et al.

2016. Neuroendocrine differentiation in breast carcinoma: clinicopathological features and outcome. Histopathology. 68: 422-32.

7. Jeon, C.H., S.M. Kim, M. Jang, B.L. Yun, H.S. Ahn, and S.W. Kim, et al. 2014.Clinical and radiologic features of neuroendocrine breast carcinomas. J Ultrasound Med. 33: 1511-8.

8. Cloyd, J.M., R.L. Yang, K.H. Allison, J.A. Norton, T. Hernandez-Boussard, and I.L. Wapnir. 2014.Impact of histological subtype on long-term outcomes of neuroendocrine carcinoma of the breast. Breast Cancer Res Treat. 148: 637-44.

9. Rovera, F., M. Lavazza, S. La Rosa, A. Fachinetti, C. Chiappa, and M. Marelli, et al. 2013.Neuroendocrine breast cancer: retrospective analysis of 96 patients and review of literature. Int J Surg. 11 Suppl 1: S79-83.

10. Wang, J., B. Wei, C.T. Albarracin, J. Hu, S.C. Abraham, and Y. Wu. 2014.Invasive neuroendocrine carcinoma of the breast: a population-based study from the surveillance, epidemiology and end results (SEER) database. BMC Cancer. 14: 147.

11. Graca, S., J. Esteves, S. Costa, S. Vale, and J. Maciel. 2012.Neuroendocrine breast cancer. BMJ Case Rep. 2012.

12. Ishida, M., T. Umeda, H. Abe, T. Tani, and H. Okabe. 2012. Neuroendocrine carcinoma of the breast with a mucinous carcinoma component: A case report with review of the literature. Oncol Lett. 4: 2932.

13. Wei, B., T. Ding, Y. Xing, W. Wei, Z. Tian, and F. Tang, et al. 2010.Invasive neuroendocrine carcinoma of the breast: a distinctive subtype of aggressive mammary carcinoma. Cancer. 116: 4463-73.

14. Rovera, F., P. Masciocchi, A. Coglitore, S. La Rosa, G. Dionigi, and M. Marelli, et al. 2008.Neuroendocrine carcinomas of the breast. Int J Surg. 6 Suppl 1: S113-5.

15. Lopez-Bonet, E., M. Alonso-Ruano, G. Barraza, A. Vazquez-Martin, L. Bernado, and J.A. Menendez. 2008.Solid neuroendocrine breast carcinomas: incidence, clinico-pathological features and immunohistochemical profiling. Oncol Rep. 20: 1369-74.

16. van Krimpen, C., A. Elferink, C.A. Broodman, W.C. Hop, A. Pronk, and M. Menke. 2004.The prognostic influence of neuroendocrine differentiation in breast cancer: results of a long-term follow-up study. Breast. 13: 329-33.

17. Adegbola, T., C.E. Connolly and G. Mortimer. 2005.Small cell neuroendocrine carcinoma of the breast: a report of three cases and review of the literature. J Clin Pathol. 58: 775-8.

18. Oberg, K. 2001.Chemotherapy and biotherapy in the treatment of neuroendocrine tumours. Ann Oncol. 12 Suppl 2: S111-4.

19. Angarita, F.A., J.L. Rodriguez, E. Meek, J.O. Sanchez, M. Tawil, and L. Torregrosa. 2013.Locallyadvanced primary neuroendocrine carcinoma of the breast: case report and review of the literature. 
World J Surg Oncol. 11: 128.

20. Inno, A., G. Bogina, M. Turazza, L. Bortesi, S. Duranti, and A. Massocco, et al. 2016.Neuroendocrine Carcinoma of the Breast: Current Evidence and Future Perspectives. Oncologist. 21: 28-32.

21. Li, S., J. Zhao, L. Zhu, F. Su, and K. Chen. 2017.Development and validation of a nomogram predicting the overall survival of stage IV breast cancer patients. Cancer Med. 6: 2586-2594.

22. Chen, J., A. Fang, M. Chen, Y. Tuoheti, Z. Zhou, and L. Xu, et al. 2018.A novel inflammation-based nomogram system to predict survival of patients with hepatocellular carcinoma. Cancer Med. 7 : 5027-5035.

23. Paiva, C.E., B. Paiva, P.N. de Paula, D.D. Preto, C.Z. de Oliveira, and S. Yennurajalingam, et al. 2018.Development and validation of a prognostic nomogram for ambulatory patients with advanced cancer. Cancer Med.

24. Chen, S., X. Li, H. Lv, X. Wen, Q. Ding, and N. Xue, et al. 2018.Prognostic Dynamic Nomogram Integrated with Inflammation-Based Factors for Non-Small Cell Lung Cancer Patients with Chronic Hepatitis B Viral Infection. Int J Biol Sci. 14: 1813-1821.

25. Fu, Y.P., Y. Yi, J.L. Huang, C.Y. Jing, J. Sun, and X.C. Ni, et al. 2017.Prognostic Nomograms Stratify Survival of Patients with Hepatocellular Carcinoma Without Portal Vein Tumor Thrombosis After Curative Resection. Oncologist. 22: 561-569.

26. Li, X.H., H. Chang, B.Q. Xu, Y.L. Tao, J. Gao, and C. Chen, et al. 2017.An inflammatory biomarkerbased nomogram to predict prognosis of patients with nasopharyngeal carcinoma: an analysis of a prospective study. Cancer Med. 6: 310-319.

27. Zhou, Z.R., C.C. Wang, X.J. Sun, Z.Z. Yang, X.X. Chen, and Z.M. Shao, et al. 2018.Prognostic factors in breast phyllodes tumors: a nomogram based on a retrospective cohort study of 404 patients. Cancer Med. 7: 1030-1042.

28. Kramer, A.A. and J.E. Zimmerman. 2007.Assessing the calibration of mortality benchmarks in critical care: The Hosmer-Lemeshow test revisited. Crit Care Med. 35: 2052-6.

29. Makretsov, N., C.B. Gilks, A.J. Coldman, M. Hayes, and D. Huntsman. 2003.Tissue microarray analysis of neuroendocrine differentiation and its prognostic significance in breast cancer. Hum Pathol. 34: 1001-8.

\section{Figures}




\section{Figure 1}

Points

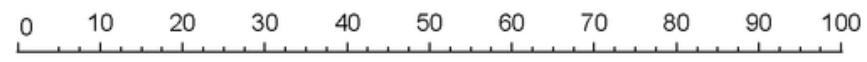

Age

$\begin{array}{lllllllllllllll}100 & 95 & 90 & 85 & 80 & 75 & 70 & 65 & 60 & 55 & 50 & 45 & 40 & 35 & 30\end{array}$

PR

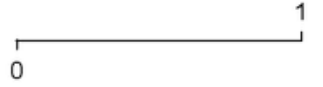

N Stage

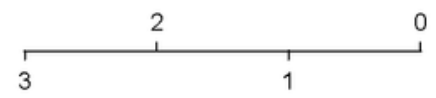

Total Points

\begin{tabular}{llllllllll}
\hline 0 & 20 & 40 & 60 & 80 & 100 & 120 & 140 & 160 & 180
\end{tabular}

5-year OS

$\begin{array}{llllll}0.1 & 0.20 .30 .40 .50 .6 & 0.7 & 0.8 & 0.9\end{array}$

\section{Figure 1}

Five-year overall survival nomogram for patients with NEBC. In order to use the nomogram, the value of each single patient is situated on each variable axis, and a line is drawn up to calculate the number of points received for every variable value. To use the nomogram, the value of an individual patient is located on each variable axis, and a line is drawn up-ward to calculate the number of points received for 
each variable value. The points were summed up together to obtain the total points and 5-year OS can be estimated the total points of each patient.

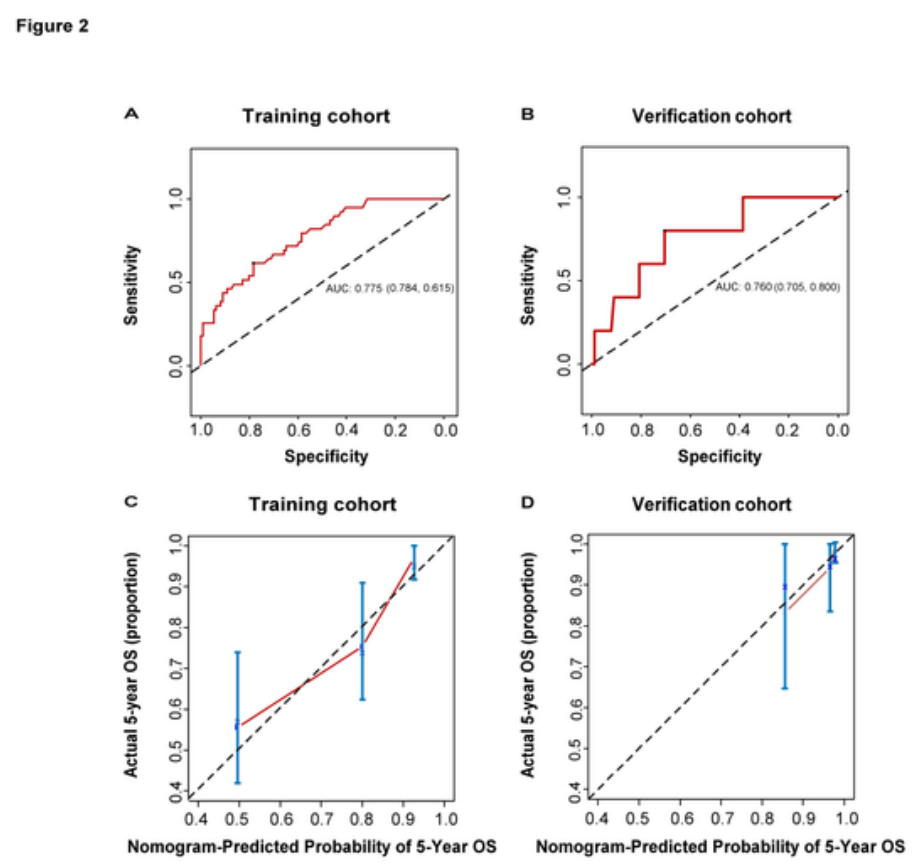

Figure 2

Discrimination and calibration of the nomogram in training and verification cohorts. Great prediction performance was observed in receiver operating characteristic (ROC) curves for discrimination of both the training $(A)$ and verification sets(B). Calibration plots of survival probability for 5 years also suggested 
pretty good consistency between the predicted 5-year OS and actual observed OS in the training(C) and verification cohort(D).

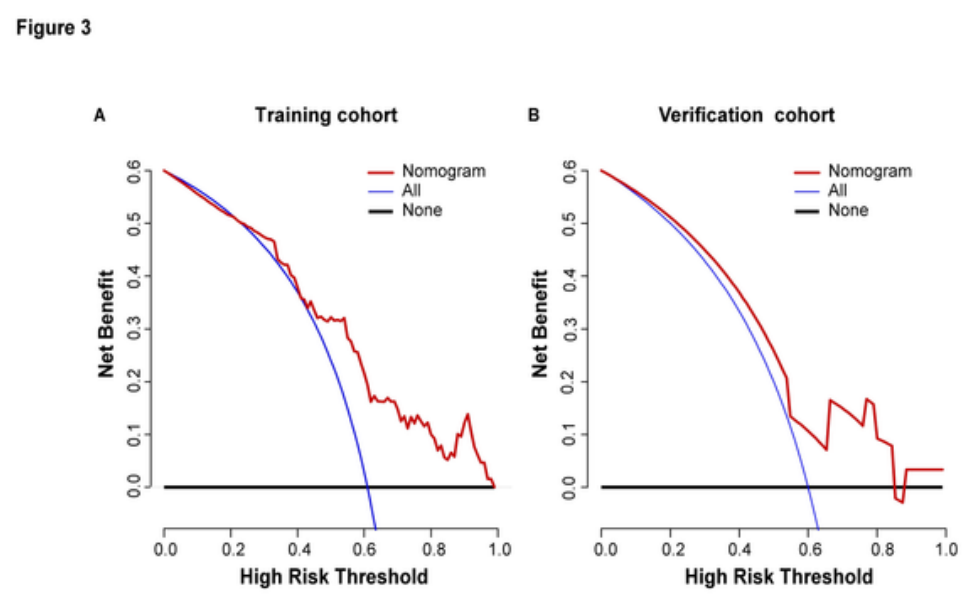

\section{Figure 3}

Decision curve analysis (DCA) for 5-year survival in training (A) and verification cohorts (B). The clinical net benefit, expressed by the $Y$-axis, was calculated by adding the benefits (true positive) and subtracting the harm (false positive). The hypothesis that no patient will experience the event is represented by 
horizontal solid black lines, while the solid blue lines represent the hypothesis that all patients will die or relapse. The solid red lines in both sets indicate good net benefit of nomogram across a range of high risk threshold. 